\title{
An Integrated Flow Analysis System for the Recalculation of Hydraulic Machinery Bladings
}

\author{
H. HAAS, N. RIEDEL, CH. WATZELT, A. FERNANDEZ and R. SCHILLING \\ Institute and Laboratory of Hydraulic Machinery and Plants Technical University of Munich, FRG
}

(Received August 9, 1995)

\begin{abstract}
The present paper deals with the recalculation of hydraulic machinery bladings. By combining numerical tools for the geometrical description of the bladings, mesh generation, different CFD flow models (Q3D Euler, 3D Euler, Navier-Stokes) and post-processing a highly efficient integrated flow analysis system is obtained.

The capability of the developed software package is shown by recalculating water turbine runners of different specific speeds, a semi-axial and a radial centrifugal impeller. The results of the flow computations are compared to each other and to existing measurements.
\end{abstract}

Key Words: Flow analysis; cfd codes; hydraulic machinery; experimental results; numerical errors

\section{INTRODUCTION}

$\mathrm{T}$ he accurate and fast analysis of the flow within already existing and/or just designed bladings is still an important task with respect to the optimization of hydraulic machinery components.

First an accurate geometry tool is needed for the description of the three-dimensional blade shapes and for the generation of arbitrary sections.

Then, based on the geometrical description of the blade surfaces the meshes needed for the flow computations have to be generated either geometrically or numerically. Finally, the numerical flow analysis may be carried out on the basis of a quasi or full 3D approach supposing inviscid or viscous flow. The flow model to be applied depends on the type of blading, the flow being accelerated or decelerated, and on the accuracy required for the flow analysis.

In order to reduce the time needed for the recalculation of the flow through a blading and for optimization of its geometry as well and to increase the reliability of the flow analysis an integrated flow analysis system has been developed (Fig. 1). This powerful numerical sysiem consists of three main modules, i.e. the geometry module, the geometrical and numerical mesh generation and finally the CFD-codes. Abreviations for the developed
CFD-codes are: Q3DEF-Q3D Euler fast, Q3DE-Q3D Euler, 3DE-3D Euler, 3DNS-3D Navier-Stokes.

\section{INTEGRATED FLOW ANALYSIS SYSTEM}

The developed flow analysis tool combines CFD models of different complexity beginning with the quasi-3D Euler code over the full-3D Euler code up to the 3D Navier-Stokes code. In the case of a rough estimation of the flow behaviour only a Q3D-analysis may be carried out. However, if a more detailled and accurate flow prediction is required a $3 \mathrm{D}$ computation is performed taking the Q3D-results as input data. Due to this strategy the CPU-time needed for the 3D-analysis may be reduced considerably. Furthermore, the reliability in predicting the flow pattern may be increased remarkably by comparing the numerical results obtained by a very complex 3D code with those from a relatively simple but highly reliable Q3D-code.

\section{Preparation of Geometrical Data}

To be mostly independend of geometrical data formats the analysis system takes the output data of comercial 


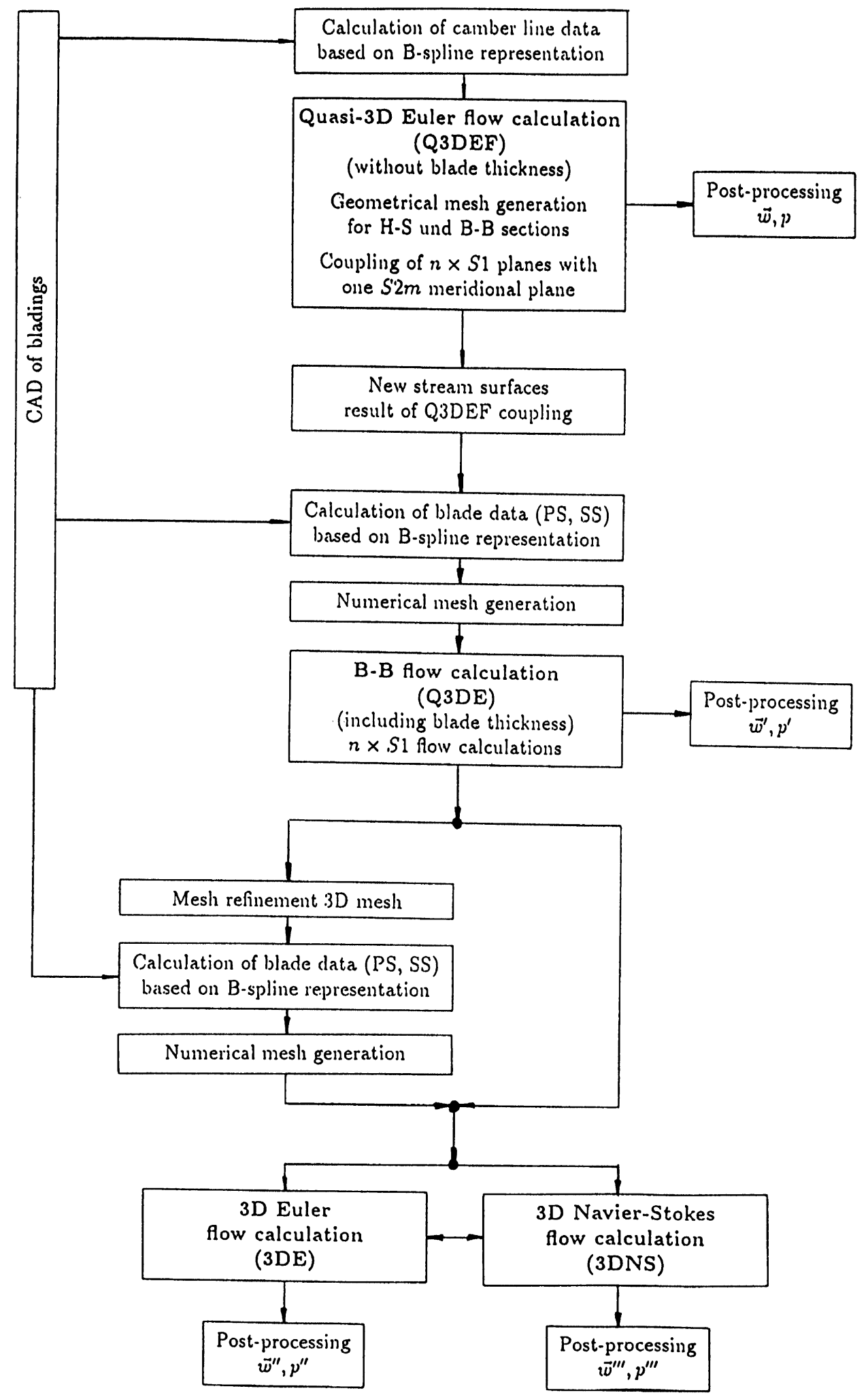

FIGURE 1 Flowchart of the flow analysis system. 
CAD systems as input. This interface to the CAD system yields the describing points of B-splines only for the blade surfaces and the meridional contour. After calculating polar coordinates $(\mathrm{r}, \varphi, \mathrm{z})$ of the blades by B-spline surface approximation the camber lines of the profiles are evaluated. Due to the B-spline representation less memory is needed to store only the describing points and a maximum geometrical accuracy is obtained.

\section{Q3D Flow Analysis}

Following a variation of the theory of $\mathrm{Wu} / 1 /$ the $3 \mathrm{D}$ flow field solution may be computed by iterating between only one representative meridional plane and a number of axisymetric blade to blade flow surfaces (Fig. 2).

To get a fast first impression of the flow behaviour the flow analysis may be performed neglecting the blade thickness. The effect of the blade thickness is taken into account by displacement factors in the meridional and blade to blade flow calculations.

This approach reduces the numerical effort considerably without a significant loss of accuracy, i.e. by using only a geometrical mesh generation in both $2 \mathrm{D}$ planes (Fig. 3) and consequently simplifying the metric coefficients of the B-B flow equation.

The basic equations describing the inviscid but rotational Q3D-flow may be derived by introducing stream functions $\psi$ satisfying the corresponding continuity equation.

$$
\begin{aligned}
& B-B \quad w_{m}=\frac{1}{r \delta} \frac{\partial \psi}{\partial \varphi}, w_{\varphi}=-\frac{1}{\delta} \frac{\partial \psi}{\partial m} \\
& \left(\frac{r}{\delta} \frac{\partial \psi}{\partial m}\right)+\frac{\partial}{\partial \varphi}\left(\frac{1}{r \delta} \frac{\partial \psi}{\partial \varphi}\right)=2 w r \sin \alpha \\
& H-S \quad c_{r}=\frac{1}{r t} \frac{\partial \psi}{\partial z}, c_{z}=-\frac{1}{r t} \frac{\partial \psi}{\partial r} \\
& \frac{\partial}{\partial r}\left(\frac{1}{r t} \frac{\partial \psi}{\partial r}\right)+\frac{\partial}{\partial z}\left(\frac{1}{r t} \frac{\partial \psi}{\partial z}\right)=\lambda
\end{aligned}
$$

where $\delta$ means the stream sheet thickness and $t$ the displacement factor in the meridional flow.

The vorticity function $\lambda$ which depends on the blade angles, the swirl of the flow and the reduced total pressure $p_{g}^{*}$ (rothalpy) may be deducted by introducing the components of blade forces in the corresponding momentum equations. Finally it may be written in the following form:

$$
\lambda=\frac{1}{r}\left\{\frac{\partial \vartheta}{\partial r} \cot \beta_{S}^{r}+\frac{\partial \vartheta}{\partial z} \cot \beta_{S}^{z}\right\}+\frac{r t}{\rho} \frac{\partial p_{g}^{*}}{\partial \psi}
$$

where $\vartheta$ denotes the swirl of the absolute flow and $\beta_{s}^{r}, \beta_{s}^{z}$ the blade angles in the $\mathbf{r}=$ const, $\mathbf{z}=$ const planes.

Introducing body-oriented coordinates and applying Gaussian's integral theorem second order node-centered finite volume schemes may be derived $/ 2 /$.
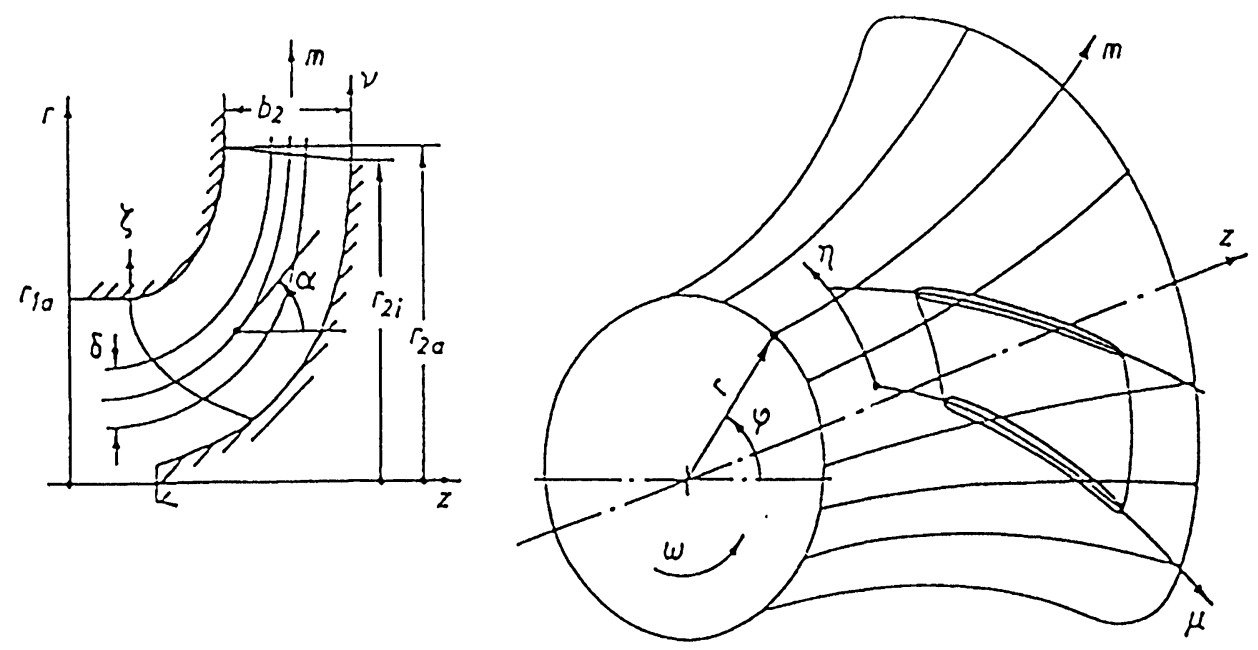

FIGURE 2 Q3D Euler flow model with body oriented coordinate system. 


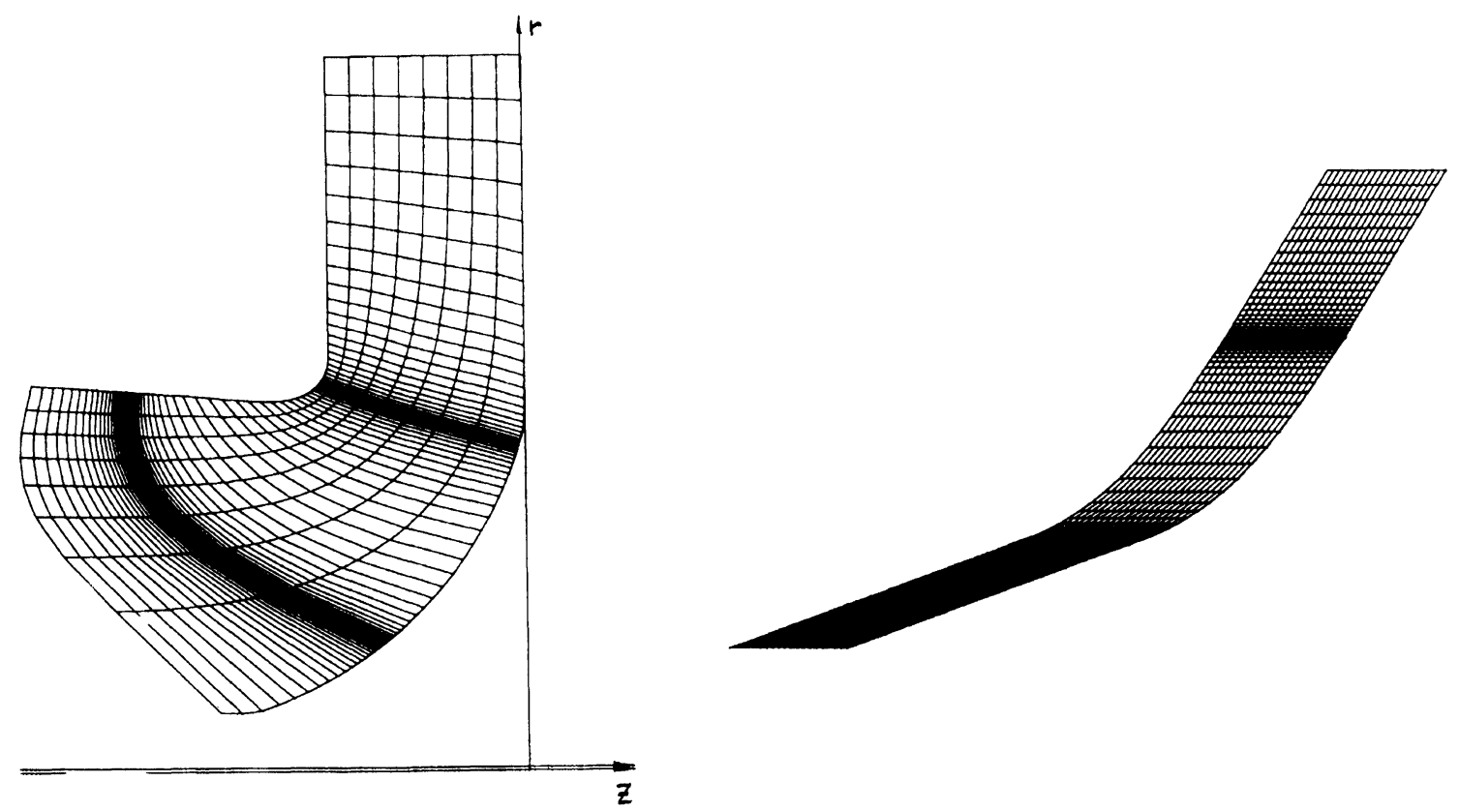

FIGURE 3 Geometrically generated meshes for H-S and B-B flow calculation.

\section{B-B Flow Analysis}

To get more accurate velocity and pressure distributions along the blades, i.e. for discussion of leading edge cavitation, a B-B flow calculation including the effect of blade thickness may be performed on the stream surfaces obtained from the Q3D iterations carried out before. This full B-B code uses basically the same algorithm as the simplified B-B code but needs more storage capacity and CPU-time, because the metric coefficients are now two dimensional arrays and numerical mesh generation has to be carried out on $\mathrm{n} \times \mathrm{S} 1$ flow surfaces.

\section{Numerical Mesh Generation on B-B Surfaces}

Following the theory of Thompson at al. /3/ and applying the Steger-Sorensen algorithm /4/ the numerical mesh generation consists of a set of two PDEs written in body-oriented coordinates. This PDEs are solved by a node-centered finite difference scheme. Connecting the generated B-B meshes, a 3D mesh is obtained with a mesh refinement to the solid boundaries and to leading and trailling edge (Fig. 4).

\section{D Flow Analysis}

The algorithm used is based on the method of artificial compressibility of Chorin /5/. Introducing the derivative of static pressure with respect to time into the continuity equation

$$
\begin{aligned}
\frac{\partial}{\partial t}\left(\frac{p}{\rho_{0}}\right)+a^{2} \cdot \operatorname{div} \vec{c}=0 ; & \vec{c}=\vec{c}(u, v, w) \\
& a=\text { artificial speed of sound } \\
& \rho_{0}=\text { constant density of the } \\
& \text { fluid }
\end{aligned}
$$

a system of hyperbolic partial differential equations is obtained. Reaching steady-state solutions, the time derivative vanishes and the divergence of the incompressible flow is satisfied being $\operatorname{div} \vec{c},=0$.

The resulting incompressible Navier-Stokes equations can be written in cartesian coordinates for a rotating system with absolute velocities as follows

$$
\begin{aligned}
& \frac{\partial}{\partial t} \iiint_{V} \vec{q} d V+\iint_{\partial V}\left(\overline{\bar{f}}_{e^{\prime}}-\overline{\bar{f}}_{v}\right) \cdot \vec{n} \mathrm{dA}+ \\
& \iint_{\partial \mathrm{V}} \overline{\bar{f}}_{e} \cdot \vec{n} \cdot \mathrm{A}+\iiint_{V} \vec{g} d V=0
\end{aligned}
$$

where $\vec{q}$, the source term $\vec{g}$, the convective flux tensors $\overline{\bar{f}}_{e} "$ and $\overline{\bar{f}}_{e}$ " and the viscous Term $\overline{\bar{f}}_{v}$ mean:

$$
\vec{q}=\left[\begin{array}{c}
\frac{p}{\rho_{0}} \\
u \\
v \\
w
\end{array}\right] \vec{g}=\left[\begin{array}{c}
0 \\
-\omega v \\
\omega u \\
0
\end{array}\right]
$$




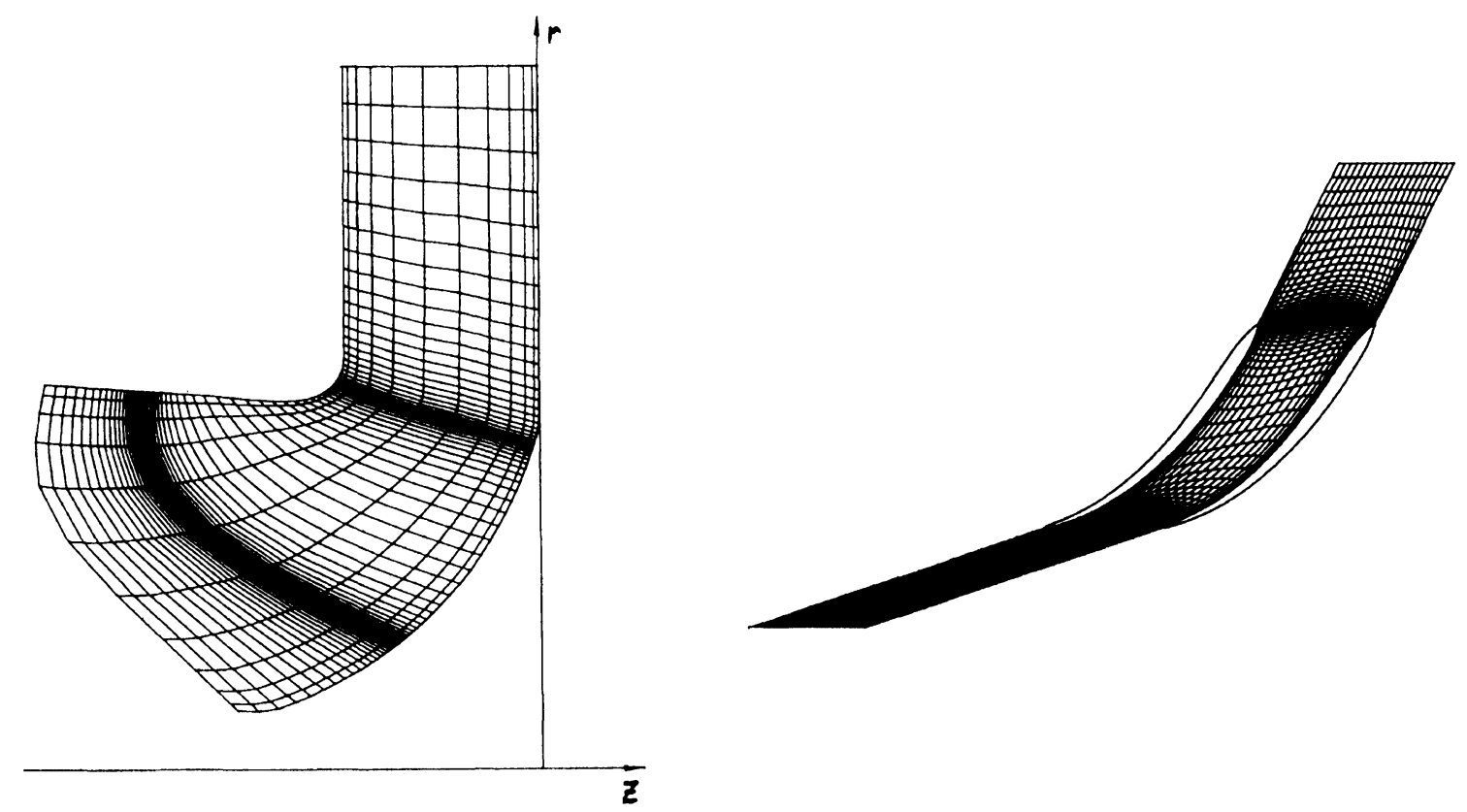

FIGURE 4 Mesh refinement at meridional mesh and numerical generated B-B mesh.

$$
\overline{\bar{f}}_{v}^{*}=\frac{1}{v}\left[\begin{array}{c}
0 \\
\frac{\partial u}{\partial x} \mathbf{i}_{\mathrm{x}}+\frac{\partial \mathrm{u}}{\partial \mathrm{y}} \mathbf{i}_{\mathrm{y}}+\frac{\partial \mathrm{u}}{\partial \mathrm{z}} \mathbf{i}_{\mathrm{z}} \\
\frac{\partial \mathrm{v}}{\partial \mathrm{x}} \mathbf{i}_{\mathrm{x}}+\frac{\partial \mathrm{v}}{\partial \mathrm{y}} \mathbf{i}_{\mathrm{y}}+\frac{\partial \mathrm{v}}{\partial \mathrm{z}} \mathbf{i}_{\mathrm{z}} \\
\frac{\partial \mathrm{w}}{\partial \mathrm{x}} \mathbf{i}_{\mathrm{x}}+\frac{\partial \mathrm{w}}{\partial \mathrm{y}} \mathbf{i}_{\mathrm{y}}+\frac{\partial \mathrm{w}}{\partial \mathrm{z}} \mathbf{i}_{\mathrm{z}}
\end{array}\right]
$$$$
\overline{\bar{f}}_{e}{ }^{\prime}=\left[\begin{array}{c}
a^{2}\left(u \mathbf{i}_{\mathrm{x}}+\mathrm{vi} \mathbf{i}_{\mathrm{y}}+\mathrm{w} \mathbf{i}_{\mathrm{z}}\right) \\
\mathrm{u}\left(u \mathbf{i}_{\mathrm{x}}+\mathrm{vi} \mathbf{i}_{\mathrm{y}}+\mathrm{w} \mathbf{i}_{\mathrm{z}}\right)+\frac{\mathrm{p}}{\rho_{0}} \mathbf{i}_{\mathrm{x}} \\
\mathrm{v}\left(u \mathbf{i}_{\mathrm{x}}+\mathrm{vi} \mathbf{i}_{\mathrm{y}}+\mathrm{w} \mathbf{i}_{\mathrm{z}}\right)+\frac{\mathrm{p}}{\rho_{0}} \mathbf{i}_{\mathrm{y}} \\
\mathrm{w}\left(u \mathbf{i}_{\mathrm{x}}+\mathrm{vi} \mathbf{i}_{\mathrm{y}}+\mathrm{wi} \mathbf{i}_{\mathrm{z}}\right)+\frac{\mathrm{p}}{\rho_{0}} \mathbf{i}_{\mathrm{z}}
\end{array}\right]
$$$$
\overline{\bar{f}}_{e}{ }^{\prime}=\left[\begin{array}{c}
a^{2}\left(-\omega \mathrm{y} \mathbf{i}_{\mathrm{x}}+\omega \mathrm{x} \mathbf{i}_{\mathrm{y}}\right) \\
\mathrm{u}\left(-\omega \mathrm{y} \mathbf{i}_{\mathrm{x}}+\omega \mathrm{xi} \mathbf{i}_{\mathrm{y}}\right) \\
\mathrm{v}\left(-\omega \mathrm{y} \mathbf{i}_{\mathrm{x}}+\omega \mathrm{xi} \mathbf{i}_{\mathrm{y}}\right) \\
\mathrm{w}\left(-\omega \mathrm{y} \mathbf{i}_{\mathrm{x}}+\omega \mathrm{xi} \mathbf{y}_{\mathrm{y}}\right)
\end{array}\right]
$$

$$
\begin{aligned}
& \begin{aligned}
r \cdot c_{\varphi} & =\text { cons } \\
\text { Solid boundaries } \quad: \vec{w} \cdot \vec{n}=0 & \frac{\partial^{2} c_{z}}{\partial \mu^{2}}=0
\end{aligned} \\
& \text { Periodic boundaries : periodicity } \\
& \text { Inlet } \\
& \text { : total pressure } ; c_{r} \\
& \begin{array}{l}
r \cdot c_{\varphi} \\
\frac{\partial^{2} c_{z}}{\partial \mu^{2}}=0
\end{array} \\
& \text { Outlet }
\end{aligned}
$$

convective flux tensor generated only by the speed of rotation and may be integrated analytically $/ 6 /$.

After discretization a second-order cell-centered finite volume scheme is obtained which is solved by means of an explicit four step Runge-Kutta procedure. For stability reasons damping terms of second and fourth order are introduced.

The numerical time integration is performed satisfying the following boundary conditions:

Here $\mu$ means the body oriented coordinate following the Here $w$ denotes the speed of rotation (pumps: $\omega>0$, turbines: $\omega<0$ ). $\overline{\bar{f}}_{e}$ " is defined as that part of the 


\section{BASIC INVESTIGATIONS OF THE NUMERICAL ACCURACY}

To check the accuracy of the developed Euler code some basic investigations have been carried out taking the axial cascade of Gostelow $/ 7 /$ as a test case. The numerically determined pressure distribution may be compared with the analytical solution. Fig. 5 shows the pressure coefficient plotted versus blade length $c_{p}(x / l)$. Using a numerical mesh of $268 \times 78$ grid points an excellent coincidence between numerical and analytical solution has been achieved.

Furthermore, the influence of the number of grid points on the numerical errors, i.e. mass flux as well as circumferentially averaged values of reduced total pressure and swirl, has been investigated. Fig. 6 clearly shows that all numerical errors are decreasing with increasing number of grid points down to a constant value of about $1 \%$.

The averaged error of the reduced total pressure occurs at the leading edge and remains constant downstream to the outlet which is an expected characteristic of a conservative Euler formulation. For the mass flux a similar behaviour is obtained. These errors are induced by the geometrical insufficiency of the H-mesh at the leading edge and by the special treatment of the artificial viscosity terms at the solid boundaries.

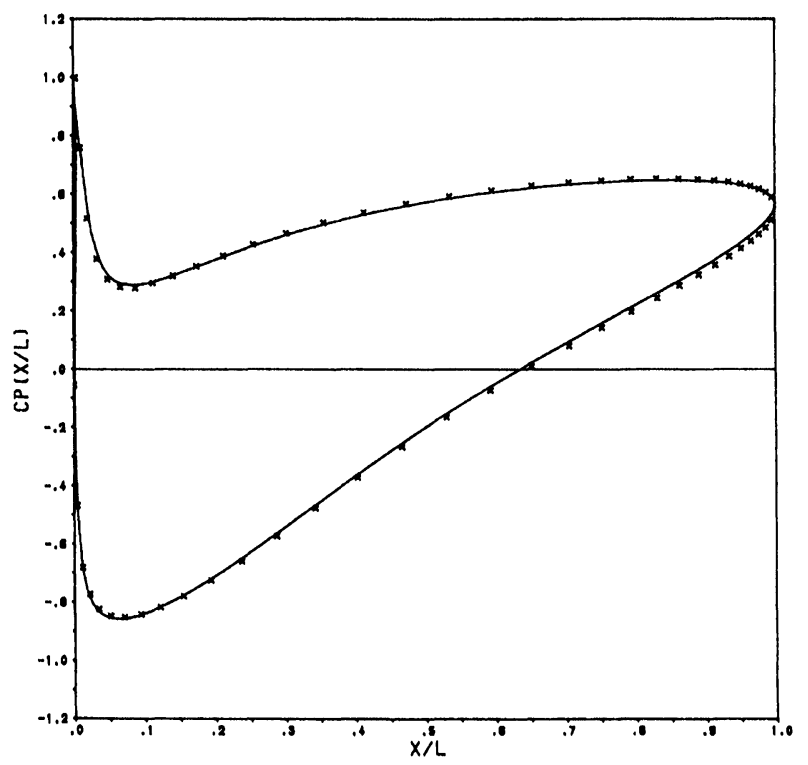

FIGURE 5 Pressure coefficient of axial Gostelow cascade ( - analytical solution, $\times$ Euler solution).

\section{RESULTS}

\section{Recalculation of Francis Turbine Runners}

The capability of the developed flow analysis system may be demonstrated analyzing three francisturbine runners with different specific speeds $\left(n_{q}=26,60\right.$ and $951 / \mathrm{min}$ ). Fig. 7 shows the distribution of static pressure along the pressure and suction side of the blading and the distribution of the circumferentially averaged swirl from inlet to outlet. All calculations have been performed for the point of best efficiency and three flow models are compared to each other (Q3DEF, Q3DE and 3DE).

Generally, a very good agreement may be observed between the three flow models applied for the flow analysis, exept for high specific speeds where three dimensional flow effects become significant. Analyzing runners or impellers having a specific speed higher than about $n_{q} \approx 801 / \mathrm{min}$ only a $3 \mathrm{D}$ code will yield accurate
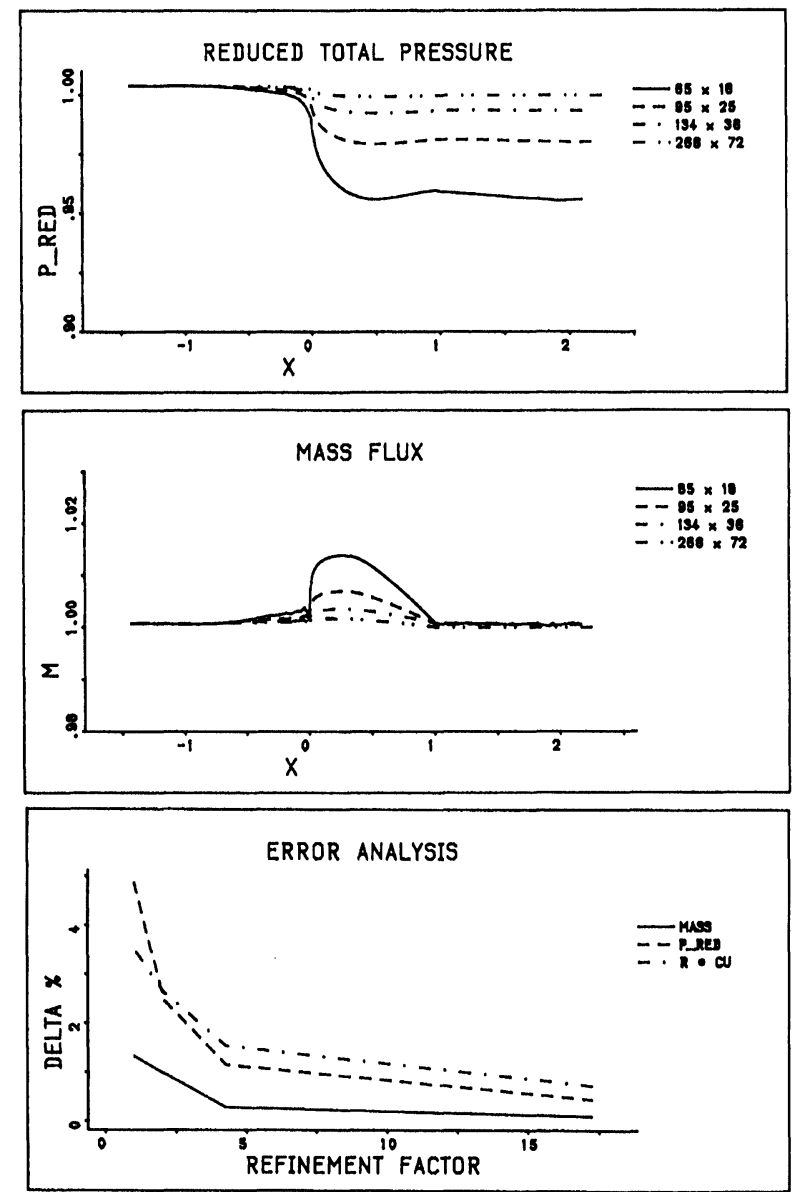

FIGURE 6 Error analysis of reduced total pressure, mass flux and $\operatorname{swirl}\left(r \times c_{u}\right)$. 

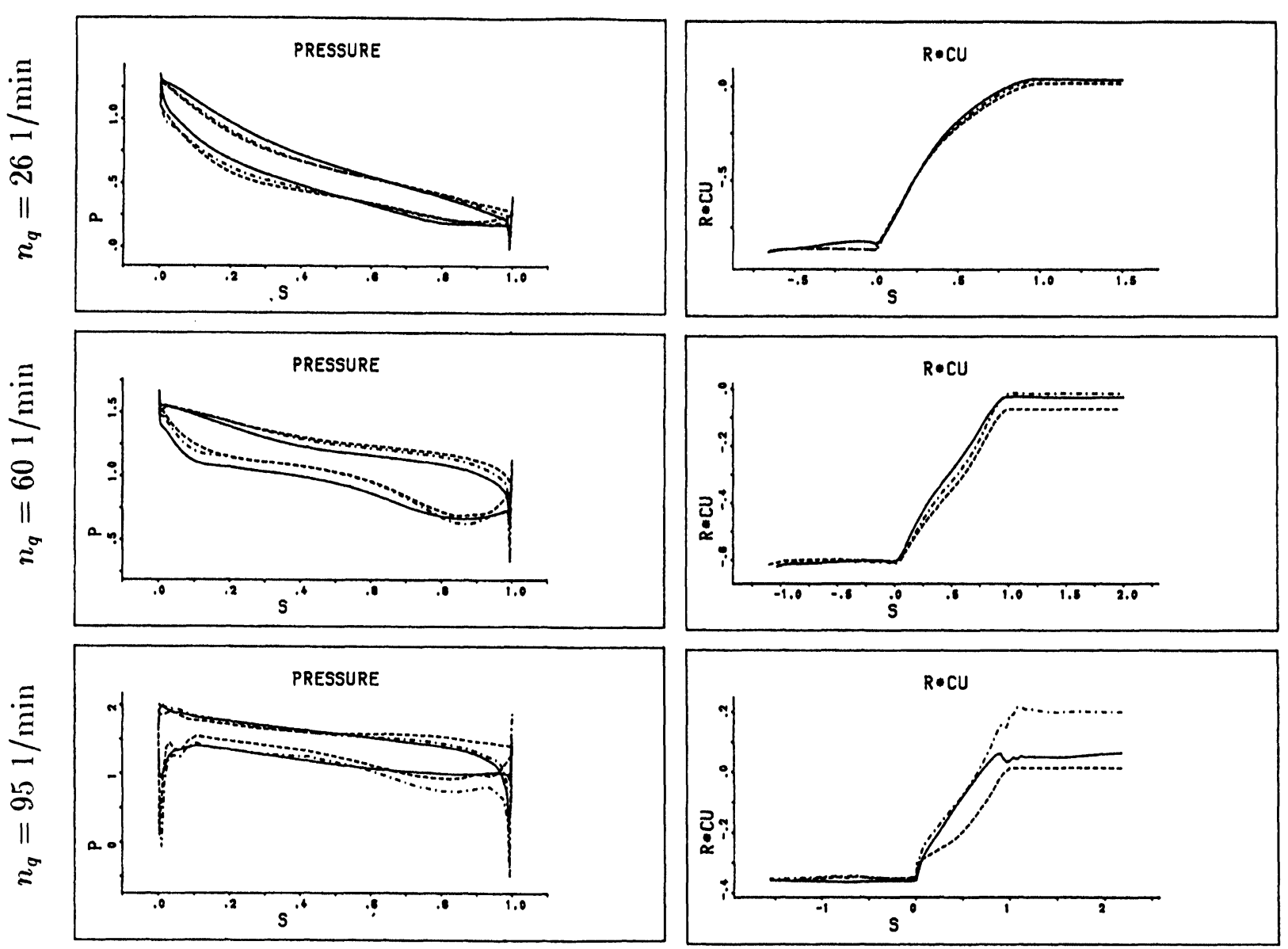

FIGURE 7 Pressure distribution on the blading and circumferentially averaged swirl of francis turbine runners with specific speeds of $n_{q}=26$, 60 and 95 1/min (-. Q3DEF, - - Q3DE, - E3D).

and reliable results. Nevertheless the relatively simple but very fast $\mathrm{Q} 3 \mathrm{DEF}$ procedure shows the same tendency concerning the pressure distribution, i.e. it allows a fast and relatively reliable insight of the flow behaviour. The ratio of CPU-time needed by the Q3DEF, Q3DE and $3 \mathrm{DE}$ code for the same number of grid points is 1:2:10, i.e. a whole Q3DEF iteration takes about $5 \mathrm{~min}$ CPUtime for a mesh with $70 \times 25 \times 9$ grid points on an IBM Risc 6000/550 workstation with a performance of about 25 Mflops.

\section{Recalculation of SHF Impeller}

In the next step the radial impeller of the Société Hydrotechnique de France (SHF) has been analyzed and the numerical results compared with varius LDV measurements of INSA, Lyon $/ 7,8 /$.

First, the pressure distributions have been computed for the best point $Q=Q_{N}$, where suction side cavitation has been observed along the leading edge from hub to shroud (Fig. 8). The comparison of computed and

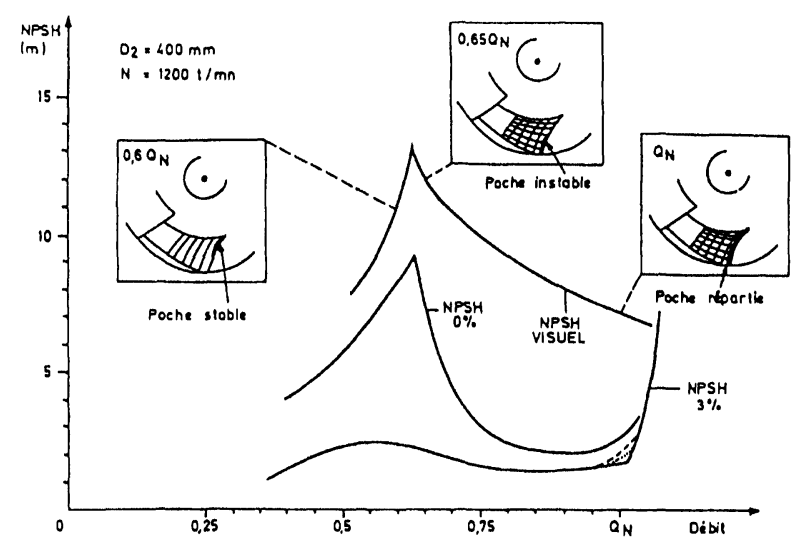

FIGURE 8 Cavitation behaviour of SHF impeller at different points of operation. 


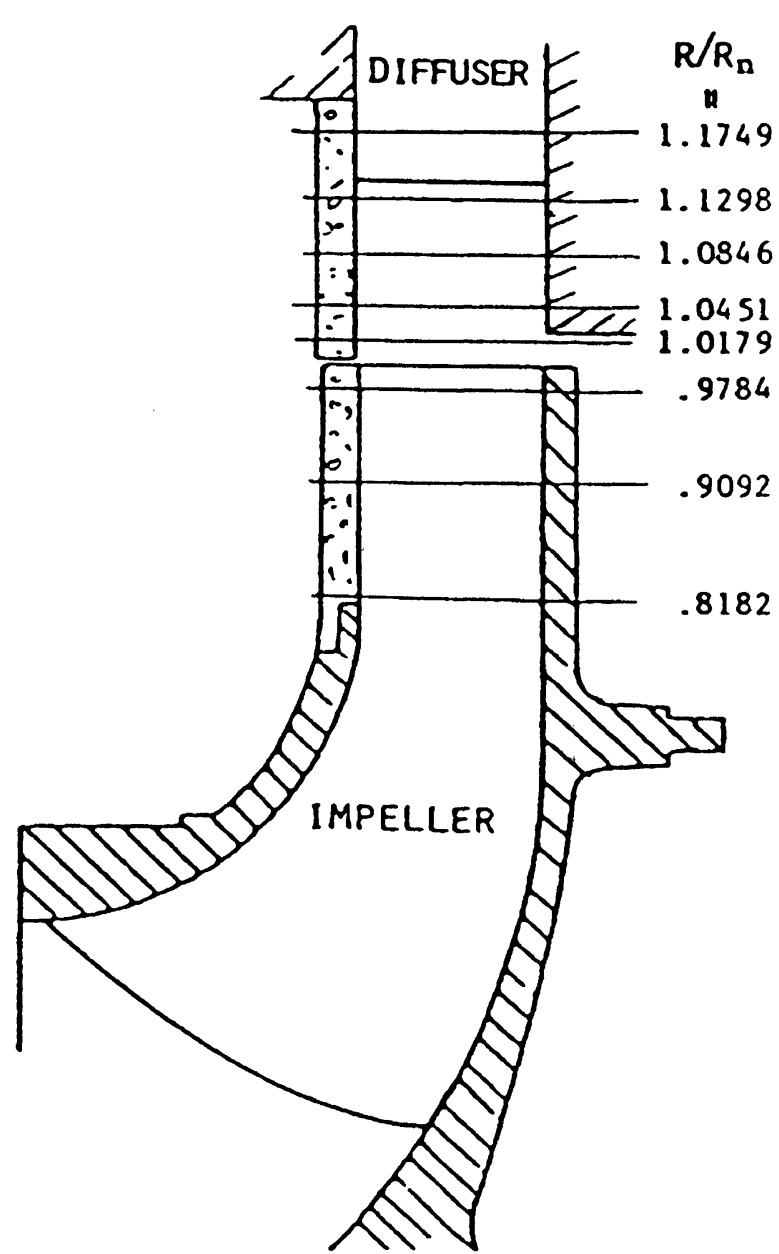

FIGURE 9 Meridional section of SHF impeller.

observed cavitation behavior shows a very good agreement. The same level of the computed suction side pressure peaks also indicates suction side cavitation along the whole leading edge (Fig. 10).

Further measurements have been made near the outlet of the impeller. Fig. 11 shows the normalized radial and tangential velocity distributions $c_{r}$ and $c_{u}$ between shroud and hub at three different radial locations (Fig. 9). Here the results of the 3D Euler computations are compared with measurements of INSA and calculations of a finite element Navier-Stokes code (N3S) developed in the Research and Development Division of Electricité de France. It is shown that the inviscid 3D Euler computations are relatively close to the Navier-Stokes computations and also to the measurements at all radii.

\section{Recalculation of NEL Impeller}

As another test case the semi-axial NEL Mark 1 impeller with a specific speed of $n_{q}=1201 / \mathrm{min}$ has been recalculated /10/. LDA measurements in front of and behind the impeller (s. Fig. 12: planes UR and 13) can be taken as a further check of the accuracy of the developed 3D Euler-code. The comparison of numerically predicted and measured velocity distributions $c_{m}(N)$ shows a rather good agreement exept near hub and shroud where the measured velocities are going to zero whereas the inviscid Euler code a priori does not satisfy this boundary condition. However, the change of velocity gradient from the inlet to the outlet of the impeller can be predicted by an inviscid flow model (Fig. 13).

Furthermore, the measurements detected a throughflow vortex at mid-chord (Fig. 12: plane 10) being induced not only by the moving casing but also by the coriolis forces shown in Fig. 14

\section{CONCLUSIONS}

Applied to turbine runners and pump impellers of different specific speeds the presented flow analysis system has proven to be a very efficient tool. It may be applied for the recalculation as well as for the optimum design of hydraulic machinery bladings. Combining CFD codes of different complexity first the CPU-time needed for one

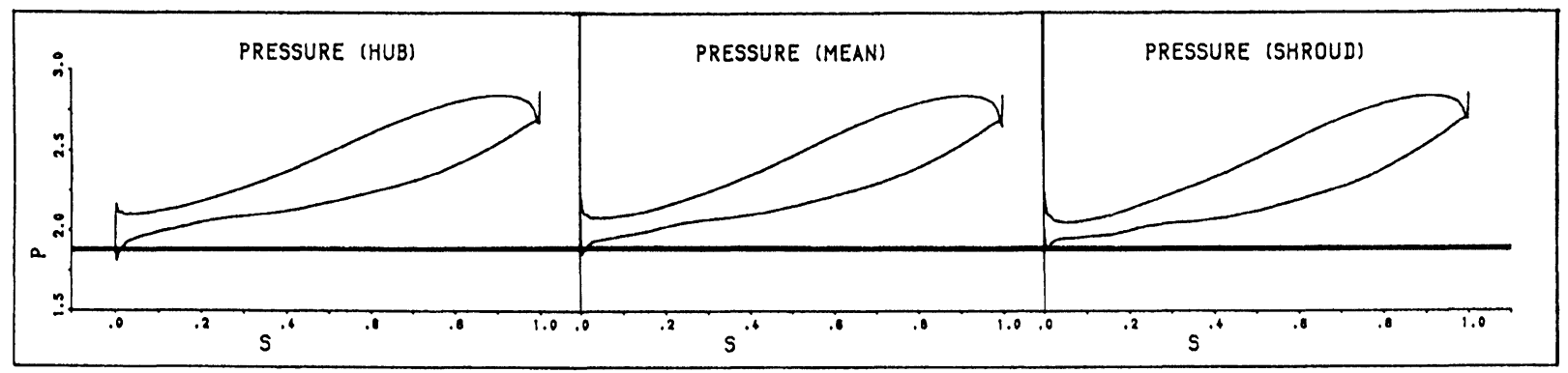

FIGURE 10 Pressure distribution along the blading of SHF impeller. 

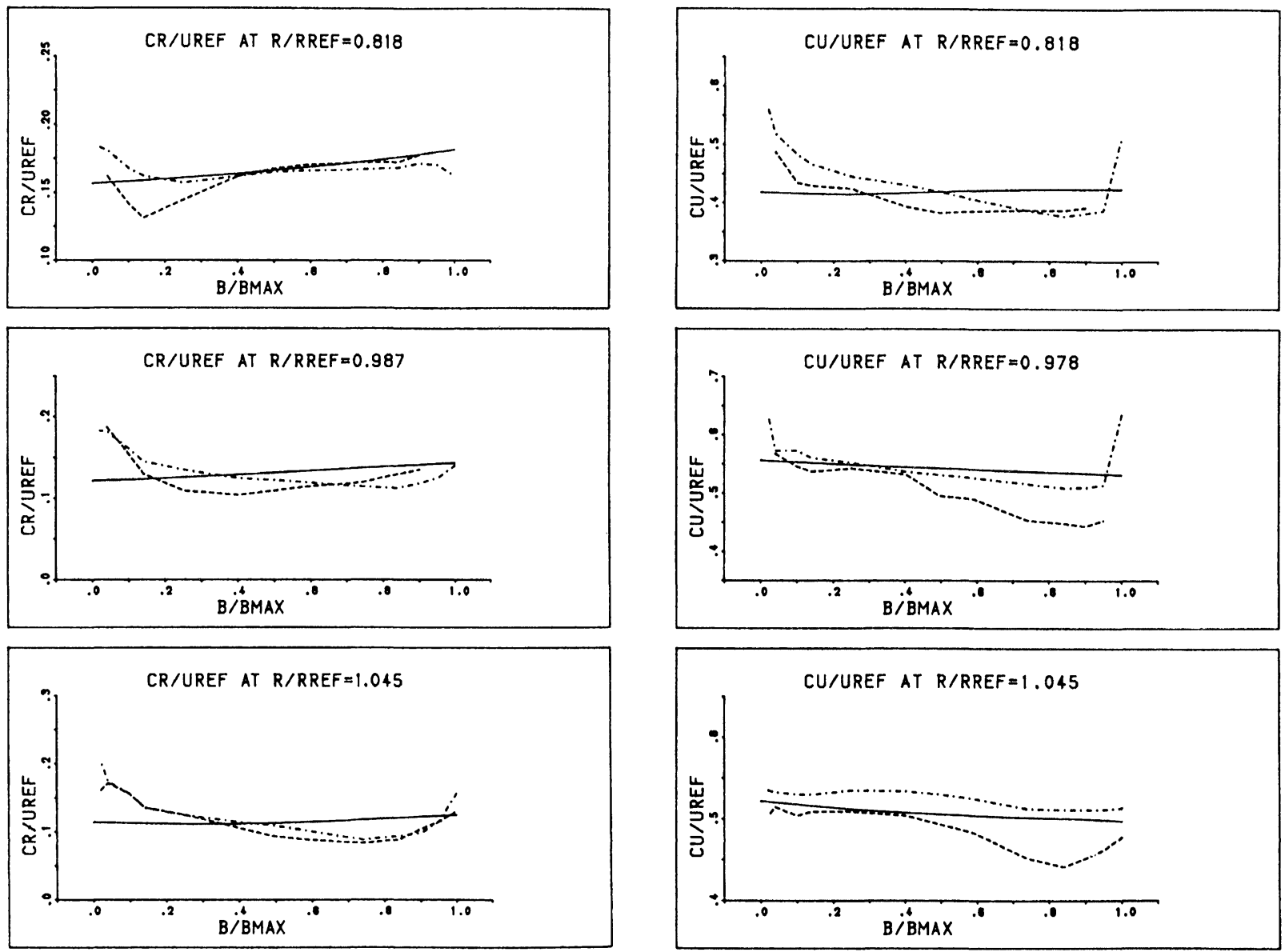

FIGURE 11 Radial and tangential velocity distribution. Comparison of results of the Euler code ( $-3 D E)$ with a Navier-Stockes code $(\cdot \cdots$ N3S) and measurements (---INSA).

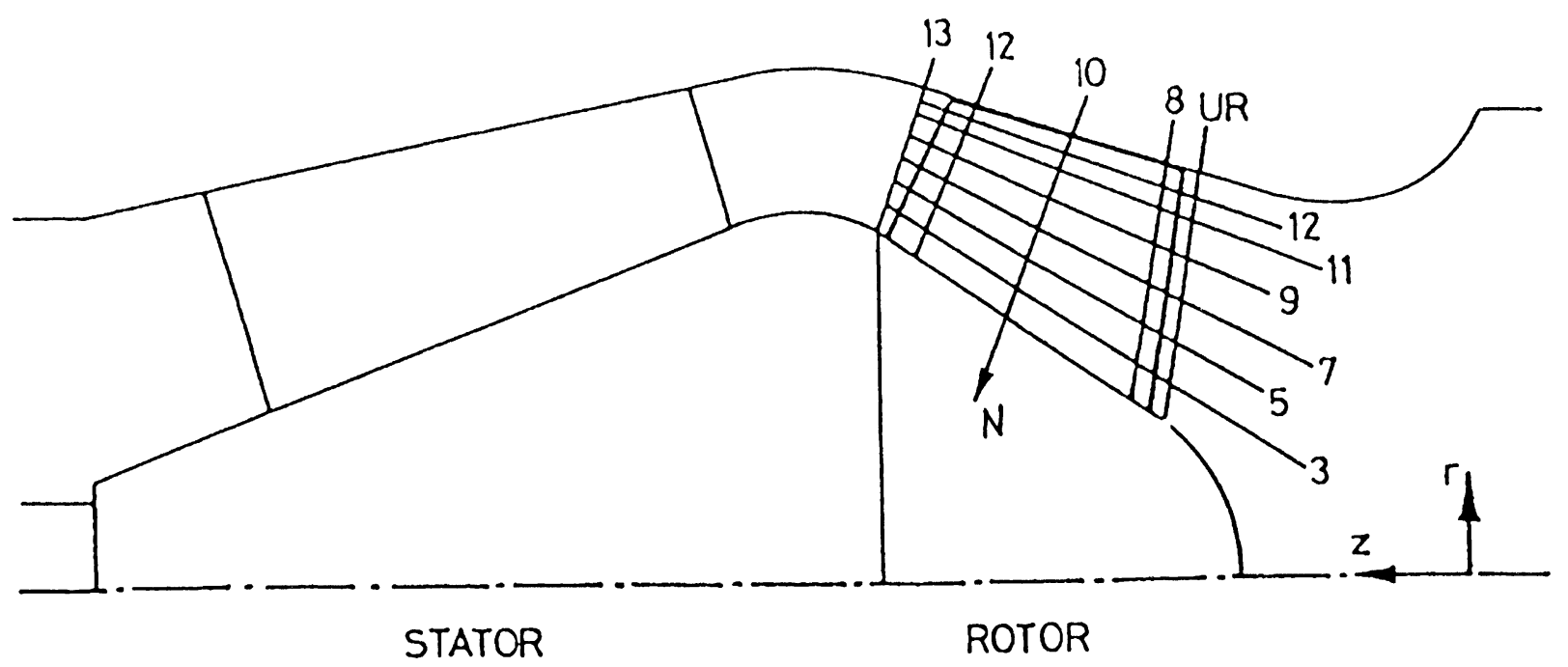

FIGURE 12 Meridional section of NEL impeller. 

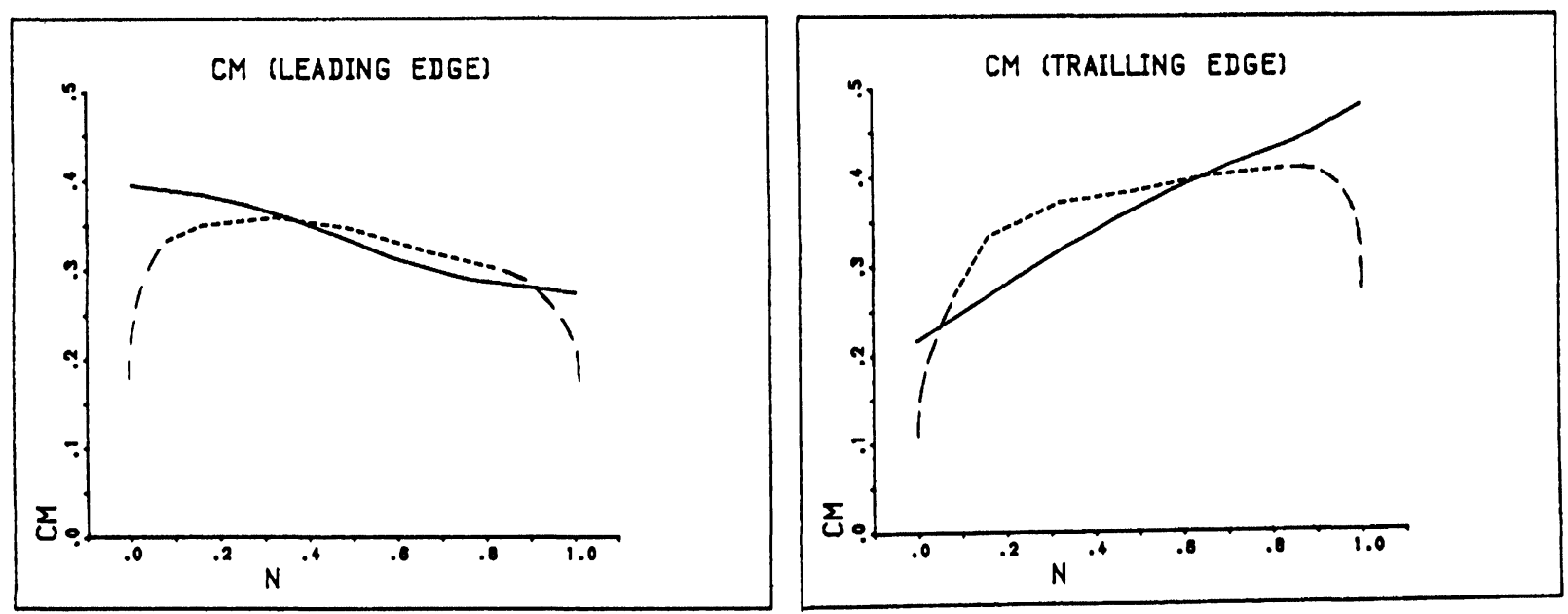

FIGURE 13 Velocities normal to LDA measuring planes in front of and behind the impeller. Comparison of 3D Euler solutions - with measurements ....

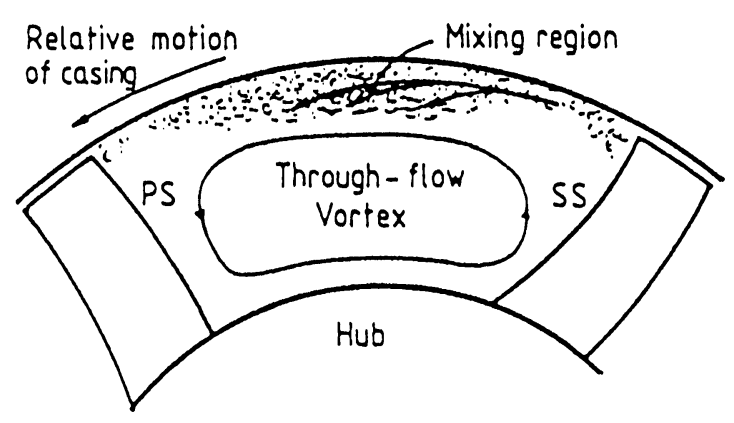

Measurement

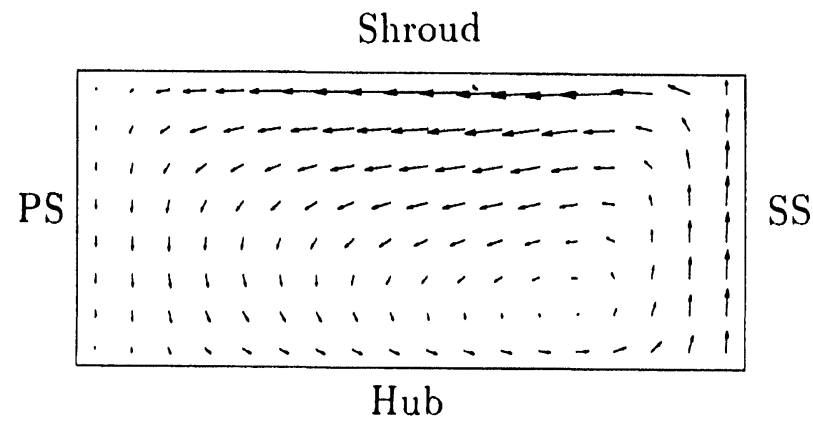

3D Euler

FIGURE 14 Section through rotor blade passage at mid chord (plane 10).

recalculation can be shortened considerably and second the reliability in interpreting the computed results may be improved.

\section{Literature}

Wu, C. H., A general theory of the $3 D$ flow in subsonic and supersonic turbomachines of axial, radial and mixed flow type. NACA, TN 2604, 1952.

Schilling, R., Watzelt, C., Haas, H., Sporer, L., CFD aided design of hydraulic machinery bladings. 9th Conference on Fluid Machinery, Budapest, 1991.

Thompson, J. F., Martin, C. W., Automatic numerical generation of body-fitted curvilinear coordinate system for field of any number of arbitrary two-dimensional bodies. J. Comp. Phys. 15, 1974.

Steger, J. L., Sorensen, R. L., Automatic mesh-point clustering near aboundary in grid generation with elliptic partial differential equations. J. Comp. Phys. 33, 1979.
Chorin, A. J., A numerical method for solving incompressible viscous flow problems. J. Comp. Phys. 2, pp. 12-26, 1967.

Kroll, N., Berechnung von Stroemungsfeldern um Propeller und Rotoren im Schwebeflug durch die Loesung der Euler-Gleichungen. Forschungsbericht DLR, DLR-FB 89-37, 1989.

Gostelow, J. P., Cascade aerodynamics. Pergamon Press, Oxford, 270 pp., 1984.

Combes, J. F., Calcul par elements finis de l'ecoulement $3 D$ turbulent dans une pompe centrifuge. 77th Synp., AGARD-PEP, 1991.

Combes, J. F., Rieutord, E., Numerical and experimental analysis of the flow in a centrifugal pump at nominal and partial flow rate. ASME paper, 92-GT-284, 1992.

Carey, C., Fracer, S. M., Rachman, D., Wilson, G., Studies of the flow of air in a model mixed-flow pump by laser doppler anemometry, Part 2: Velocity measurements within the impeller. National Engineering Laboratory, Nel Report No 699, 1985. 

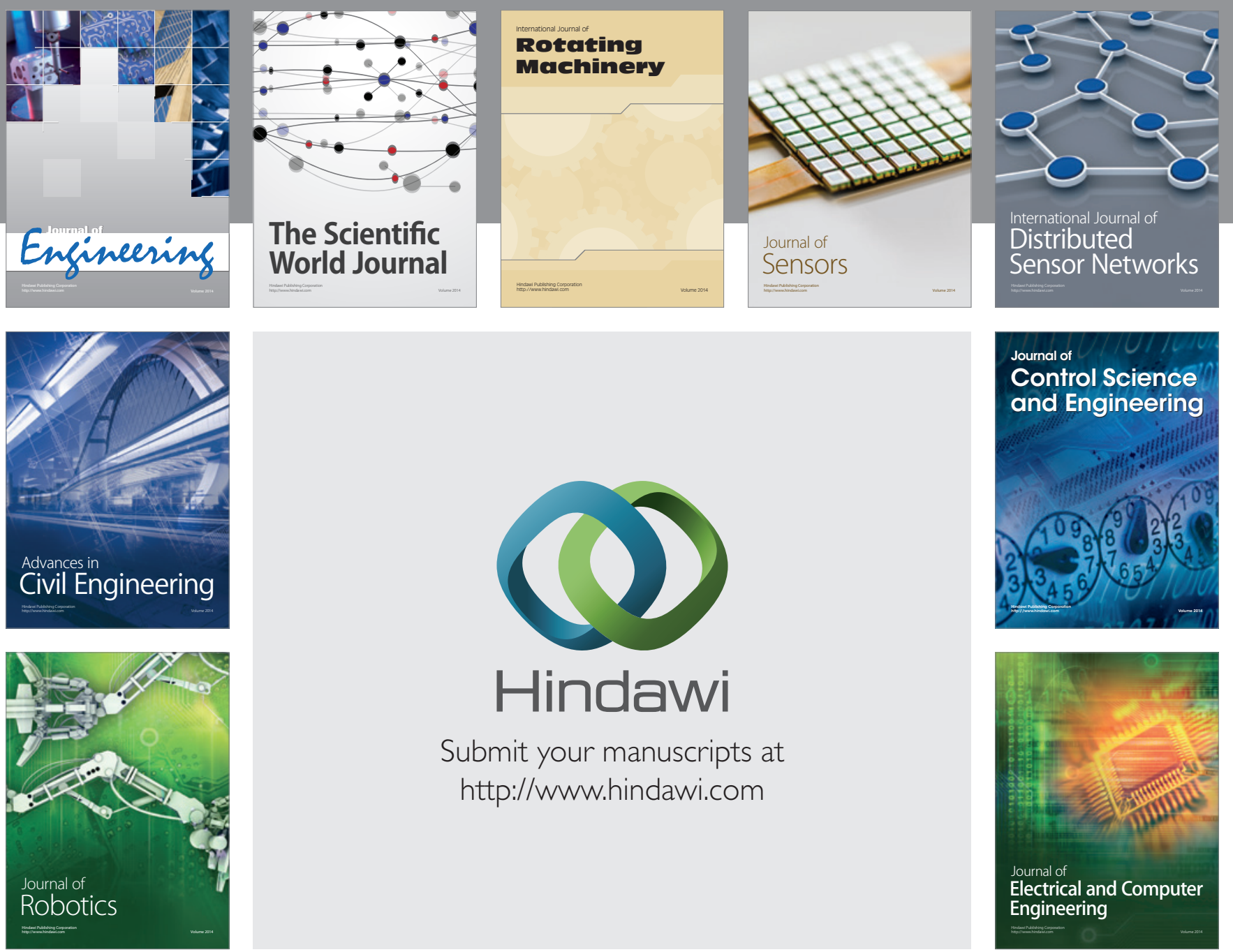

Submit your manuscripts at

http://www.hindawi.com
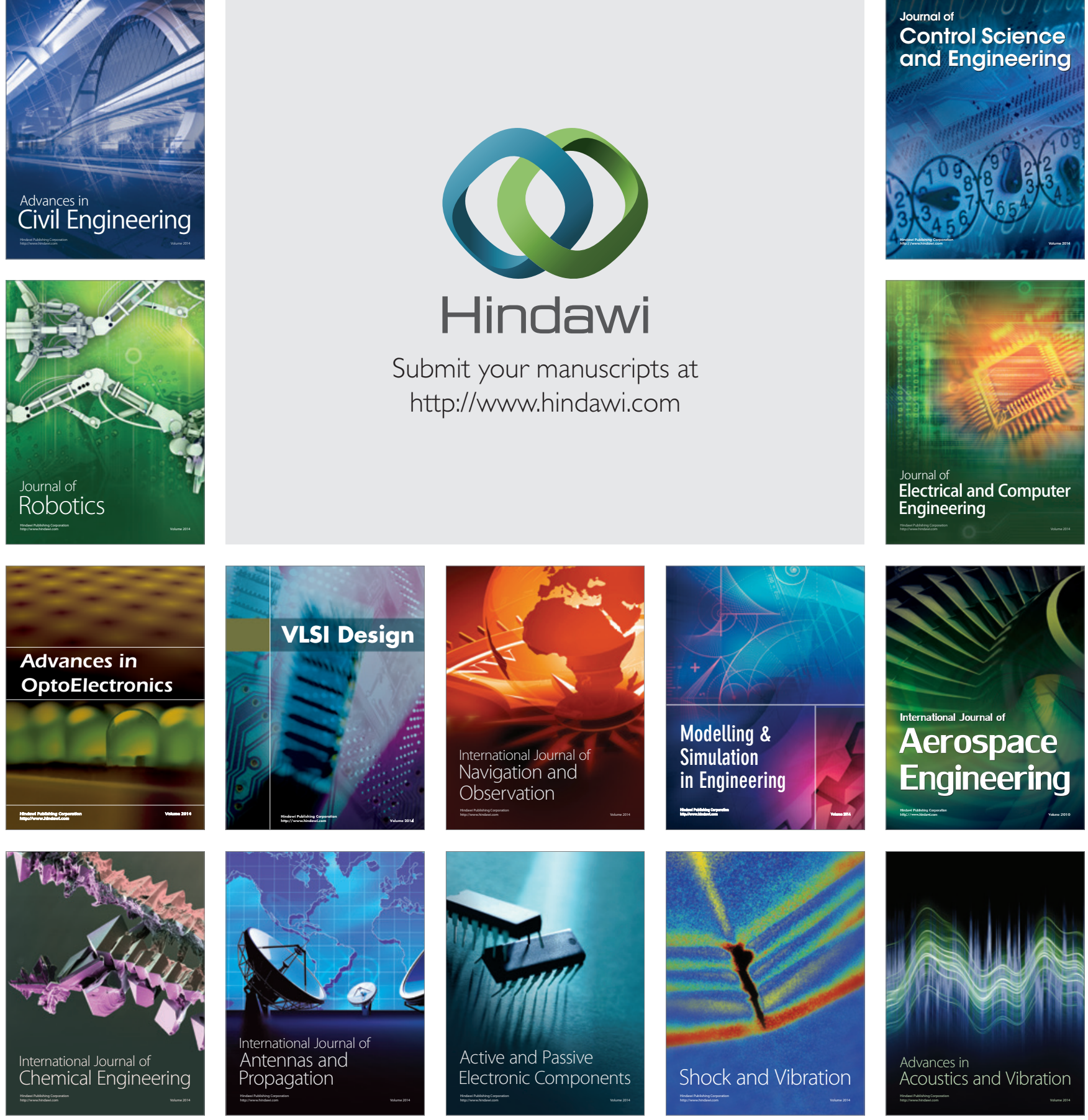